\title{
Verortungen und Abgrenzungen der Intuition
}

In den folgenden beiden Kapiteln geht es darum, die bisher entwickelte Intuitionskonzeption für Anwendungen fruchtbar zu machen. In Kap. 5 bestehen diese Anwendungen in der Verhältnisbestimmung der Intuition zu anderen mentalen Zuständen: Wie verhält sich Intuition zu Imagination und zu Formen der Irrationalität (aliefs, motivierte Irrationalität, Delusion)? In Kap. 6 folgt eine Auseinandersetzung mit der methodischen Debatte über Intuition, die in der zeitgenössischen analytischen Philosophie intensiv geführt wird. Aufgrund des zuvor entwickelten Intuitionsverständnisses erscheinen wichtige Aspekte dieser Debatte in neuem Licht.

\subsection{Intuition und Imagination}

Die Untersuchung des Verhältnisses zwischen Intuition und Imagination verdient eine ausführliche Behandlung, da die Imagination einerseits entscheidend für die Bildung mentaler Modelle ist, also für die Ausgangspunkte der Intuition, andererseits aber auch Zielpunkt der Intuition sein kann.

\subsection{1 >Intuition < und >Imagination< in philosophiegeschichtlicher Dynamik} Der Begriff >Imagination< leitet sich vom lateinischen imago ab, was ursprünglich die Fähigkeit bezeichnet, Bilder zu haben oder herzustellen. Verwandt damit ist der Begriff imitatio, welcher den mimetischen, abbildenden Aspekt der Imagination hervorhebt. ${ }^{268}$ Verbreitete deutsche Ausdrücke, die $>$ Imagination $<$ entsprechen, sind $>$ Einbildung $<$ bzw. $>$ Einbildungskraft $<$ und Vorstellung' bzw. >Vorstellungskraft $<.{ }^{269}$ Auf Platon zurückgehend wird - vor allem innerhalb des Rationalismus - Imagination als mindere Form der Erkenntnis gewertet, da sie als Nachbildung des sinnlich Empfangenen noch weiter entfernt von derWahrheit als die Sinneswahrnehmung selbst ist. Damit ergibt sich ein aus heutiger Sicht überraschend starker Gegensatz zwischen Imagination und Intuition, da die Intuition durch diese Positionen als epistemisch hochrangig eingeordnet wird.

\footnotetext{
268 Vgl. Brann (1991, S. 18).

269 Vgl. Mainusch/Warning (1976, S. 217).
} 
Diese Entgegensetzung und Wertung zeigt sich deutlich bei Descartes: Im Discours de la méthode ordnet er die Imagination als Gegenpol zum Verstand ein, wodurch sie auf derselben Seite wie die Sinnlichkeit steht, aber dennoch von dieser unterschieden wird:

und deshalb scheint mir jemand, der seine Anschauung gebrauchen will, um sie [= die Ideen, C.M.] zu begreifen, genau dasselbe zu tun wie jemand, der seine Augen benutzen will, um Töne zu hören oder Gerüche zu riechen - wobei allerdings ein Unterschied noch darin besteht, dass das Sehvermögen als Sinn uns der Wahrheit seiner Objekte genauso wenig versichert wie der Geruchssinn oder das Gehör als solche, während weder unsere Anschauung noch unsre Sinne uns jemals irgendeines Dinges versichern können, wenn unser Verstand nicht dabei eingreift. ${ }^{270}$

Descartes bringt seine Skepsis gegenüber der Imagination als Erkenntnisquelle auch an weiteren Stellen im Discours zum Ausdruck. So insistiert er, wir dürften uns »nur von der Evidenz unserer Vernunft überzeugen lassen « und keineswegs »von unserer Anschauung « (also von der Imagination) oder »von unseren Sinnen«. Als Beispiel zur Unterstützung dieser These führt Descartes an, dass wir uns einen Löwenkopf auf dem Körper einer Ziege vorstellen können, ohne dass sich daraus auf das tatsächliche Bestehen einer solchen Chimäre schließen ließe. ${ }^{271}$ In einem Brief an Picot hält er weiter fest, dass »die Wahrheit unsere Anschauung oftmals nicht so sehr anregt, wie es die Falschheiten und Täuschungen tun.«272 Somit sind bei Descartes Intuition und Imagination, trotz der etymologischen Verwandtschaft der Begriffe, in Bezug auf deren Beitrag zur Erkenntnis weit voneinander entfernt. Der Grund dafür ist, dass Intuition eine geistige Anschauung bezeichnet, also die unmittelbare und gewisse Erkenntnis apriorischer Wahrheiten, während Imagination eine Vorstellung der bereits unsicheren Sinneseindrücke und somit eine noch unsicherere Erkenntnisquelle als diese ist.

Bei Spinoza setzt sich die unterschiedliche Wertung von Intuition und Imagination hinsichtlich der Erkenntnis fort, wie sich in der Differenzierung der Erkenntnisgattungen in der Ethica zeigt: Während imaginatio (zusammen mit opinio) entweder die direkt aus den Sinnen stammende Erfahrung, welche die Einzeldinge »verstümmelt, verworren und ohne Ordnung « darstellt, oder die durch Erinnerungen und Assoziationen aus dieser Sinneserfahrung

270 AT VI 37 - Übersetzung aus dem Französischen hier und im Folgenden von Wohlers (2011). Wohlers übersetzt imagination mit Anschauung.

271 Vgl. AT VI 39.

272 AT VIII/2 17 (Übersetzung Wohlers 2011). 
abgeleiteten Eindrücke bezeichnet, können Spinoza zufolge nur ratio und scientia intuitiva adäquate Ideen hervorbringen. ${ }^{273}$

Eine Gegenbewegung zur rationalistischen Geringschätzung der Imagination lässt sich in Kants Transzendentalphilosophie erkennen. Indem Kant die Imagination (bei ihm >Einbildungskraft $<$ ) als Erkenntnisgrundlage aufwertet, legt er den Grundstein für spätere Würdigungen der Imagination, welche sie mit Kreation assoziieren und somit zu einer entscheidenden Größe in der Ästhetik werden lassen. ${ }^{274}$ In der Kritik der reinen Vernunft führt Kant die Einbildungskraft als entscheidende Vermittlerin zwischen Sinnlichkeit und Verstand ein:

Einbildungskraft ist das Vermögen, einen Gegenstand auch ohne dessen Gegenwart in der Anschauung vorzustellen. Da nun alle unsere Anschauung sinnlich ist, so gehört die Einbildungskraft, der subjektiven Bedingung wegen, unter der sie allein den Verstandesbegriffen eine korrespondierende Anschauung geben kann, zur Sinnlichkeit; so fern aber doch ihre Synthesis eine Ausübung der Spontaneität ist, [...] so ist die Einbildungskraft so fern ein Vermögen, die Sinnlichkeit a priori zu bestimmen [...]. So fern die Einbildungskraft nun Spontaneität ist, nenne ich sie auch bisweilen die produktive Einbildungskraft, und unterscheide sie dadurch von der reproduktiven, deren Synthesis lediglich empirischen Gesetzen, nämlich denen der Assoziation, unterworfen ist, und welche daher zur Erklärung der Möglichkeit der Erkenntnis a priori nichts beiträgt $[\ldots] .{ }^{275}$

Während die reproduktive Einbildungskraft das bezeichnet, was bei den Rationalisten die ganze Imagination war, nämlich eine weitere Nachbildung des sinnlich Gegebenen, hebt Kant neu die produktive Rolle der Einbildungskraft hervor, die dadurch bedingt ist, dass »ihre Synthesis eine Ausübung der Spontaneität ist «. Wie dies zu verstehen ist, wird in weiteren Textpassagen der $\mathrm{KrV}$ sowie auch der KdU ersichtlich:

Weil aber jede Erscheinung ein Mannigfaltiges enthält, mithin verschiedene Wahrnehmungen im Gemüte an sich zerstreuet und einzeln angetroffen werden, so ist eine Verbindung derselben nötig, welche sie in dem Sinne selbst nicht haben können. Es ist also in uns ein tätiges Vermögen der Synthesis dieses Mannigfaltigen, welches wir Einbildungskraft nennen, und deren unmittelbar an den Wahrnehmungen ausgeübte Handlung ich Apprehension nenne. ${ }^{276}$

Nun gehören zu einer Vorstellung, wodurch ein Gegenstand gegeben wird, damit überhaupt daraus Erkenntnis werde, Einbildungskraft, für die

\footnotetext{
273 E II 40s2 - siehe auch oben, Kap. 2.2.

274 Vgl. Mainusch/Warning (1971, S. 217ff.).

$275 \mathrm{KrV}$ B $151 \mathrm{f}$.

276 KrV A 120; siehe außerdem A 123.
} 
Zusammensetzung des Mannigfaltigen der Anschauung, und Verstand für die Einheit des Begriffs, der die Vorstellungen vereinigt. ${ }^{277}$

Kant gesteht also der Imagination nicht bloß eine passive Rolle als Nachbildung sinnlicher Eindrücke zu, sondern lässt sie im Erkenntnisprozess aktiv und entscheidend werden: Da Kant zufolge Sinnlichkeit und Verstand gleichermaßen Quellen der Erkenntnis sind und die Anschauung unter Begriffe subsumiert werden muss, bedarf es der Imagination, die diese Subsumption leistet. Grund dafür ist die Mannigfaltigkeit der empirischen Anschauungen. Diese müssen bereits als Bündel vorliegen (daher »Synthesis des Mannigfaltigen«), damit überhaupt eine geeinte Erscheinung vorliegt, die unter Begriffe subsumiert werden kann. Kant hebt somit die Bedeutung der Imagination, verstanden als produktive Imagination, über den Bereich des Sinnlichen hinaus. Nachdem in der rationalistischen Tradition Intuition die hochgeschätzte Erkenntnisquelle mit Zugang zu apriorischer Wahrheit, Imagination demgegenüber auf das Unsichere und Sinnliche verwiesen war, wendet sich das Blatt bei Kant: Imagination wird zu einer wichtigen Grundlage der Erkenntnis, indem sie zwischen Sinnlichkeit und Verstand vermittelt, während Intuition auf den Bereich des Empirischen eingegrenzt wird.

\subsubsection{Eine Abgrenzung in Bezug auf Realitätssensitivität}

Im philosophiegeschichtlichen Wandel des Intuitions- und Imaginationsbegriffes haben sich die Abgrenzungen und Wertungen verändert, bleiben aber jeweils klar benennbar. Im Gegensatz dazu scheint in jüngerer Zeit die Abgrenzung zwischen Intuitions- und Imaginationsbegriff diffuser. Ein Bereich, in dem dies besonders auffällt, ist der Diskurs über wissenschaftliche Erkenntnisse. Exemplarisch dafür werden zunächst einige Auszüge eines populärwissenschaftlichen Werks angeführt: John Beveridges The Art of Scientific Investigation. ${ }^{278} \mathrm{Im}$ Anschluss daran soll ein Vorschlag zur Klärung des Verhältnisses zwischen Intuition und Imagination in Bezug auf die Erkenntnis skizziert werden.

Beveridge separiert die Behandlung von Imagination und Intuition, indem er diesen Konzepten je ein eigenes Kapitel widmet und darin anhand zahlreicher Beispiele auf die Bedeutung der jeweiligen Erkenntnisart für den wissenschaftlichen Erkenntnisprozess eingeht. Einleitend dazu hält er fest, dass es in beiden Kapiteln darum gehe, »how ideas originate in the mind and

277 AA V B 28; siehe außerdem B 65, B 145, B 193 f.

278 Vgl. Beveridge (1957). 
what conditions are favourable for creative mental effort «. ${ }^{279}$ Als erstes Beispiel für die Bedeutung der Imagination erwähnt Beveridge den berühmt gewordenen Bericht Kekulés über dessen Entdeckung des Benzolrings: Kekulé habe, ermüdet nach langem und eher erfolglosem Arbeiten, die Augen geschlossen und sei eingenickt. Daraufhin habe er im Traum die Figur einer Schlange imaginiert, die sich in den eigenen Schwanz beißt und sei dadurch auf die Lösung des Benzolrings gekommen. Dieses Beispiel stellt Beveridge in eine Reihe weiterer Beispiele, aus denen er insgesamt den Schluss zieht: »Pictorial analogy can play an important part in scientific thinking. « ${ }^{280}$

Hier liegt also Imagination im Sinne einer bildlichen Vorstellung vor (imagery). ${ }^{281}$ Doch zugleich scheint in diesen Beispielen Intuition involviert zu sein: So ist es durchaus angemessen zu sagen, dass Kekulé durch Intuition zur Benzolring-Lösung gekommen sei. Gerade durch das in den vorigen Kapiteln entwickelte nichtpropositionale Intuitionsverständnis wird dies weiter plausibilisiert, denn es wird dadurch nicht gefordert, dass Kekulé eine $>$ Intuition, dass $\mathrm{p}<$ hatte, sondern, dass ihm ein mentales Modell bekannt war, welches die Grundlage einer Intuition $\mathrm{NP}_{\mathrm{N}}$ wurde. Dies scheint hier durchaus gegeben, denn die für Intuition charakteristischen phänomenalen Merkmale der Unmittelbarkeit und Gewissheit stellten sich gemäß Kekulés Bericht in Bezug auf seine Benzolring-Imagination ein. Die Imagination kann also als Manifestation (im Sinne einer imagery) einer entsprechenden Intuition $_{\mathrm{NP}}$ bezeichnet werden.

Um sich weiter davon zu überzeugen, dass Kekulés Erkenntnis eine intuitive ist, kann man sich die beschriebene Situation nochmals vor Augen führen: Kekulé hatte sich vor dem Einnicken ausgiebig mit der Materie beschäftigt. Dadurch ist anzunehmen, dass ihm alle Fakten zur Verfügung standen, die für die Benzolring-Lösung benötigt werden. Als Experte verfügte Kekulé also bestimmt über ein mentales Modell bzw. mehrere mentale Modelle der Sachlage, und der Halbschlaf diente ihm dazu, dieses mentale Modell (unwillentlich) zu verfestigen. Entweder bereits in diesem Dämmerzustand oder spätestens nach dem Aufwachen ereignete sich das »Ablesen« des mentalen Modells, wodurch Kekulé die Intuition ${ }_{\mathrm{NP}}$ gewann. Diese wurde ihm schließlich in Form der Benzolring-Imagination (also einer imagery) zugänglich. Nun

\footnotetext{
279 Beveridge (1957, S. 53).

280 Vgl. Beveridge (1957, S. 56).

281 McGinn folgend verwende ich für die auf Sinneswahrnehmung basierende Vorstellung den Begriff imagery, um diese von der kognitiven Imagination zu unterscheiden (vgl. McGinn 2004, S. 128f.). Der Unterschied zwischen imagery und kognitiver Imagination wird in einem Beispiel Descartes' gut ersichtlich: So kann ich mir im kognitiven Sinn ein Tausendeck vorstellen, nicht aber im Sinne der imagery (vgl. AT VII 57).
} 
könnte man sich fragen: Warum gerade in Form einer Imagination? Die einfache Antwort, die hier genügen soll, lautet: Warum nicht? Wenn wir keine Schwierigkeiten damit haben, Intuitionen ${ }_{\mathrm{NP}}$ als propositionale $>$ Intuitionen, dass $\mathrm{p}$ < oder intuitive Überzeugungen manifestiert zu sehen, sollten wir auch Imaginationen als solche Manifestationen zulassen, wie auch andere Erkenntnisse in imaginativer Form zum Ausdruck kommen können. Diese Erklärung von Kekulés Erkenntnisprozess ist plausibler als einige zunächst naheliegend scheinende Alternativen: So fällt Kekulé nicht aus dem Nichts eine Imagination zu, der man einen unerklärbaren Erkenntniswert zusprechen müsste. Vielmehr ist anzunehmen, dass die Imagination des Benzolrings in ursächlichem Zusammenhang mit seinen zuvor ausgiebig betriebenen Studien steht. ${ }^{282}$

Die Vermutung, dass bei Kekulé die Intuition im Zentrum steht, wird durch Beveridges eigene weitere Ausführungen unterstützt. So präsentiert Beveridge in seinem Kapitel zur Intuition ähnliche Beispiele wie dasjenige Kekulés. Diesen weiteren Beispielen ist gemeinsam, dass Forscher (u.a. Poincaré, Gauß, Loewi) eine Einsicht hatten, die mit den phänomenalen Merkmalen der Unmittelbarkeit und Gewissheit gekennzeichnet ist. Daraus schließt Beveridge hinsichtlich der Intuition:

The most characteristic circumstances of an intuition are a period of intense work on the problem accompanied by a desire for its solution, abandonment of the work perhaps with attention to something else, then the appearance of the idea with dramatic suddenness and often a sense of certainty. ${ }^{283}$

Frappant ist, wie gut diese Beschreibung auch auf das Beispiel Kekulés passt, obwohl sich dieses in Beveridges Kapitel über die Imagination befindet. Es scheint, dass Intuition nicht unbedingt mit imagery verbunden, jedoch umgekehrt Imagination auf Intuition beruhen muss, damit von Erkenntnis gesprochen werden kann. Kekulé und alle anderen Wissenschaftler in Beveridges Beispielen kommen durch Intuition auf die entsprechende Erkenntnis: Diese kann sich als imagery anzeigen, wie es im Fall Kekulés geschehen ist, wobei aber diese Form der Imagination die Intuition nicht ersetzt, sondern auf dieser aufbaut.

282 Durchaus wäre ein anderer Fall möglich: Kekulé könnte auch spontan die BenzolringImagination gebildet haben, wach und willentlich oder im Traum. Es geht hier nicht darum zu spekulieren, was sich im Fall Kekulés tatsächlich abgespielt hat; denn dessen ungeachtet darf wohl als unkontrovers gelten, dass es Fälle gibt, auf welche die vorliegende Erklärung zutrifft.

283 Beveridge (1957, S. 73). 
Aus der Analyse des Kekulé-Beispiels lässt sich somit ein starkes Postulat für eine Differenzierung zwischen Intuition und Imagination hinsichtlich ihres Beitrags zur Erkenntnis ableiten, wie sie bereits bei den rationalistischen Positionen vorhanden war. Im Folgenden soll gezeigt werden, inwiefern sich diese Differenzierung auch innerhalb des in dieser Arbeit entwickelten Intuitionsverständnisses vornehmen lässt. Der Schlüssel zum Verständnis liegt in der unterschiedlichen Realitätssensitivität von Intuition und Imagination. Darunter ist zu verstehen, dass sich ein Subjekt seine Intuitionen nicht ausdenkt, sondern diese einen Ausschnitt der vorhandenen Welt für das Subjekt wiedergeben, was bei der Imagination nicht der Fall ist: Diese wird durch das Subjekt quasi neu erstellt.

In üblichen Fällen von Imagination geschieht diese willentlich. So kann ich mir beispielsweise willentlich vorstellen, wie ich an einer Strandbar sitze und ein kühles Getränk genieße, während die Wellen rauschen, ich das Meer rieche, das Getränk schmecke und ein bestimmtes Musikstück aus der Richtung der Bar höre. Diese Imagination entspricht nicht der Realität, wenn ich an einem regnerischen Spätherbsttag an meinem Bürotisch sitze. Der Irrealitätsgrad der Imagination lässt sich nahezu beliebig steigern: Beispielsweise kann ich mir vorstellen, ich sei ein Popstar, der in dieser Strandbar sitzt, oder mir vorstellen, ich sitze als Popstar in einer Bar auf dem Mars. Dies zeigt, dass Imagination realitäts-insensitiv ist. Sie kann sich dadurch auf reale aber auch auf irreale Situationen, Gegenstände und Sachverhalte beziehen. ${ }^{284}$

Im Unterschied zur Imagination ist Intuition realitätssensitiv. Der Gehalt unserer Intuitionen hängt von Gegebenheiten ab, was aber nicht heißt, dass Intuitionen veridisch sind, wie die Beispiele fehlgeleiteter Intuitionen zeigten. Auch ist damit nicht gemeint, dass wir alles, was wir intuitiv erfassen, für wahr halten; man vergegenwärtige sich die >Intuition, dass $\mathrm{p}<$, die am Beispiel des Mathematikers mit der Spielerfehlschluss-Intuition beschrieben wurde. Besser lässt sich die Bedeutung von >Realitätssensitivität< klären, indem daran erinnert wird, dass Intuitionen Neigungen zu Überzeugungen konstituieren. Demnach können auf der nichtpropositionalen Grundlage von Intuitionen propositionale intuitive Überzeugungen aufbauen. Entsprechendes ist bei Imaginationen nicht der Fall. Ich kann mir vorstellen, in einer Strandbar auf dem Mars zu sitzen, ohne auch nur im Geringsten die Neigung zu entsprechenden Überzeugungen auszubilden. Anders im Beispiel des intuitiven Spielerfehlschlusses: Ohne die aus dem mathematischen Wissen stammende

284 Vgl. Sartre (1980, S. 64), Wittgenstein (Z, § 621), McGinn (2004, S. 132), Gendler (2010, S. 162). 
gegenläufige Überzeugung ist nicht zu denken, wie ich - immer unter der Voraussetzung, die entsprechende Intuition wird überhaupt Grundlage einer Überzeugung - nicht die dem Spielerfehlschluss entsprechende Überzeugung ausbilden könnte (siehe Kap. 3.2).

Die Unterscheidung zwischen Intuition und Imagination hinsichtlich deren Realitätssensitivität steht mit dem in dieser Arbeit vorgelegten Intuitionsverständnis in Einklang. Gemäß diesem kommen Intuitionen durch das Ablesen vorhandener mentaler Modelle in der Typ 1-Kognition zustande. Das Vorhandensein dieser Modelle ist die Grundlage der Realitätssensitivität der Intuition, wobei hier der Realitätsbegriff relativiert werden muss: Unsere Intuitionen können auch in die Irre gehen und sich beispielsweise auf durch und durch irreale Vorstellungen beziehen. Entscheidend ist aber, dass diese Fantasiewelten bereits zuvor, auf der Ebene der mentalen Modelle, konstituiert wurden. Diese können die Realität fehlerhaft repräsentieren oder auch von Beginn an fiktional sein. Die Theorie der mentalen Modelle scheint also eine plausible Grundlage für die festgestellte Realitätssensitivität der Intuition zu bieten.

Die Unterscheidung bezüglich der Realitätssensitivität erklärt die bisherigen Befunde zu Intuition und Imagination vortrefflich. Zunächst wird dadurch klar, warum philosophische Positionen in der rationalistischen Tradition den beschriebenen großen Unterschied zwischen Intuition und Imagination hinsichtlich der Erkenntnis gemacht haben: Selbstverständlich ist nur ein realitätssensitiver mentaler Zustand dazu in der Lage, uns der Wahrheit näher zu bringen: also die Intuition und nicht die Imagination. Folgerichtig ist auch, dass die Imagination hinsichtlich der Erkenntnis noch skeptischer beurteilt wird als die Sinneserfahrung, da letztere - wenn auch nach rationalistischer Auffassung oft in die Irre gehend - mit der Intuition doch die Realitätssensitivität teilt; und kaum ein Rationalist würde sagen wollen, dass uns die Sinneserfahrung immer in die Irre führt. Die Realitätssensitivität erklärt aber auch die entgegengesetzte Bewertung von Intuition und Imagination bei Kant. Dies, da Kant aufzeigt, inwiefern auch aktive Leistungen des Subjekts für jegliche Erkenntnis zentral sind und dieser vorhergehen müssen. Gerade die Willkürlichkeit der Imagination, die sich in deren Realitäts-Insensitivität zeigt, führt bei Kant also zu deren Hochschätzung.

Letztlich ist es auch der Unterschied bezüglich der Realitätssensitivität, der zur Klärung des Unterschieds zwischen Intuition und Imagination in Erkenntnisprozessen beiträgt, wie anhand von Beveridges The Art of Scientific Investigation gezeigt wurde. Imaginationen können gerade aufgrund ihrer Realitäts-Insensitivität Start- und Zielpunkte intuitiver Erkenntnisprozesse 
sein. Startpunkte sind die Imaginationen, wenn sie mentale Modelle konstituieren, die anschließend auf Typ 1-Ebene abgelesen werden. Das Ablesen führt zur eigentlichen Erkenntnis, also der Intuition. Diese kann wiederum beliebigen Ausdruck finden; so ließen sich auch andere bildliche (allenfalls auch auditive und haptische) Imaginationen denken, welche die Benzolring-Erkenntnis quasi codieren. Zwar hat Kekulé nicht absichtsvoll den Benzolring imaginiert, doch es ist völlig plausibel, das Erkenntnissubjekt Kekulé auch in diesem Fall als aktiv zu beschreiben. Wie wir selber es sind, die im Traum Bilder imaginieren - auch wenn dies in der Regel nicht absichtsvoll geschieht - ist es auch Kekulé selber, welcher der bereits vorhandenen Intuition im Traum die Gestalt der kreisförmigen Schlange gibt.

Zusammenfassend lässt sich Imagination als eine wertvolle Ausdrucksform oder auch Grundlage der Erkenntnis verstehen, die aber von der Intuition zu unterscheiden ist. Im Einklang mit verbreiteten Verwendungsweisen des Intuitionsbegriffs und mit der Theorie der mentalen Modelle lässt sich festhalten: Intuition bezeichnet eine Erkenntnisgattung, Imagination hingegen eine Erkenntnisgrundlage oder einen Erkenntnisausdruck. Diese Unterscheidung lässt die Ausarbeitung einer Theorie der intuitiven Erkenntnis zu, die von Imaginationen ausgehen oder zu Imaginationen hinführen kann.

Die soeben gemachten Ausführungen zum Verhältnis zwischen Intuition und Imagination können erneut zur Befürchtung Anlass geben, hier sei einem ungebührlich weiten Intuitionsbegriff Tür und Tor geöffnet worden. Grund für diese Befürchtung ist insgesamt der zentrale Stellenwert, welcher der Typ 1-Kognition und generell dem Nichtpropositionalen im dargestellten Modell intuitiver Rechtfertigung gegeben wurde. Zusätzlich motiviert wird die Befürchtung des zu weiten Intuitionsbegriffes durch den postulierten Einschluss fallibler und aposteriorischer Erkenntnisse. Was, so kann man nun fragen, grenzt die Intuition von Formen der Irrationalität ab? Zwar wurde in Kap. 4 bereits besprochen, welche internalistischen sowie externalistischen Kriterien dafür notwendig sind, dass Intuitionen Gründe für Subjekte werden können. Formen irrationalen Denkens würden diese Kriterien vermutlich nicht erfüllen. Jedoch zeigen solche Merkmale der Rechtfertigung den Unterschied zwischen Intuition und anderen mentalen Zuständen eher bloß an, statt ihn selbst zu beschreiben. Daher sollen im Folgenden drei irrationale mentale Zustände von der Intuition abgegrenzt werden. Ich orientiere mich an den in der aktuellen Philosophie der Psychologie dominierenden Diskussionen und untersuche dementsprechend die Abgrenzung der Intuition von aliefs, Fällen motivierter Irrationalität (Wunschdenken, Selbsttäuschung) und Delusionen. 


\subsection{Intuition und aliefs}

Tamar Gendler hat in zwei aufeinander bezogenen Aufsätzen den alief als neuen Begriff für einen mentalen Zustand eingeführt, der sich in verschiedener Hinsicht von der Überzeugung (belief) unterscheidet und - eine Überzeugung unterstützend oder dieser zuwiderlaufend - zentralen menschlichen Erfahrungen und Handlungsweisen zugrunde liegt. ${ }^{285}$ Die Stringenz und Bedeutung des alief-Konzepts demonstriert Gendler anhand einer Vielzahl von Beispielen sowie psychologischen Forschungsergebnissen. Ein Vergleich zwischen aliefs und Intuitionen ist vor dem Hintergrund des bisher erarbeiteten Intuitionsverständnisses naheliegend, da es sich in beiden Fällen um nichtpropositionale bzw. nichtpropositional fundierte mentale Zustände handelt, die darüber hinaus weitere Gemeinsamkeiten aufweisen. Nach einer Darstellung des alief-Konzepts bei Gendler sollen die Gemeinsamkeiten und Unterschiede zur Intuition erörtert werden.

\subsubsection{Gendlers alief-Konzept}

Der alief wird von Gendler als mentaler Zustand beschrieben, der die folgenden zentralen Merkmale aufweist:

- repräsentational

- affektiv aufgeladen

- handlungsbezogen

- durch Eigenschaften im Inneren des Subjekts oder seiner Umwelt aktiviert

- automatisch

- arational

- sowohl bei Menschen als auch bei nichtmenschlichen Tieren auftretend

- eigenständiger Zustand (z.B. unabhängig von Überzeugung) $)^{286}$

Gendler erläutert die genannten Merkmale der aliefs anhand eines Beispiels:287 Am Grand Canyon wurde eine Glaskonstruktion gebaut, der sogenannte Skywalk, dessen gläserner Boden und gläserne Wände den Blick auf den hunderte Meter tiefen Abgrund freigeben. Das Begehen des Skywalk stellt für viele Besuchende eine Mutprobe dar. Ein bei solchen Konstruktionen bekanntes Phänomen ist, dass die Besuchenden zwar in der Regel der Überzeugung sind, das Begehen des Skywalk stelle keinerlei Gefahr dar, jedoch zugleich einen dieser Überzeugung entgegengesetzten Gedanken haben, dessen

285 Vgl. Gendler (2008a) und (2008b). Beide Aufsätze sind im Sammelband Gendler (2010) abgedruckt, nach dem im Folgenden zitiert wird.

286 Vgl. Gendler (2010, S. 263, 288).

287 Vgl. Gendler (2010, S. 256). 
Gehalt sich verbal etwa so umschreiben lässt: »Sehr hoch, geht sehr weit hinunter, kein sicherer Ort sich aufzuhalten! Entfernen!« Einen solchen Gedanken bezeichnet Gendler als alief.

Der alief im Skywalk-Beispiel weist alle genannten Merkmale auf: Der alief geht mit Furcht einher, ist also affektiv aufgeladen. Eine starke Handlungsbezogenheit ist ebenfalls gegeben (»entfernen!«). Die Aktivierung erfolgt im Skywalk-Beispiel durch Eigenschaften der Umwelt, nämlich durch die gläserne Konstruktion, wobei auch Eigenschaften des Subjekts eine Rolle spielen, wie etwa dessen Neigung zu Höhenangst. Der alief tritt automatisch auf, d.h. die Repräsentation erfolgt angesichts der Glaskonstruktion unweigerlich und zumindest zu einem hohen Grad - unkontrollierbar. Er ist arational, insofern sein Gehalt keinen Gründen des Subjekts zugänglich ist, d.h. weder positiv durch Gründe konstituiert wird (= nicht rational) noch negativ durch Gründe unterbunden werden kann (= nicht irrational). ${ }^{288}$ Ein alief ist in gewisser Hinsicht primitiv: Sein Auftreten ist nicht an höhere kognitive Fähigkeiten gebunden. So ist gut vorstellbar, dass sich bei Kleinkindern oder Hunden, die unter den Skywalk-Besuchenden sind, ähnliche aliefs beobachten lassen. Die Eigenständigkeit des aliefs gegenüber Überzeugungen zeigt sich im SkywalkBeispiel dadurch, dass Subjekte dem alief entgegengesetzte Überzeugungen haben können: so etwa die Überzeugung, dass der Skywalk sicher ist und sein Begehen keine Gefahr darstellt.

\subsubsection{Gemeinsamkeiten zwischen Intuition und alief}

Nach dieser Charakterisierung des aliefs lässt sich die Frage angehen, welche Gemeinsamkeiten und Unterschiede aliefs mit Intuitionen aufweisen.

Eine erste und offensichtliche Gemeinsamkeit ist die Nichtpropositionalität dieser beiden mentalen Zustände. ${ }^{289}$ Wie wir gesehen haben, können Intuitionen ganz im Typ 1-Denken verhaftet bleiben und von dort aus direkt handlungswirksam werden. Dasselbe gilt - wie am Beispiel des Skywalk offensichtlich wird - auch für aliefs. Ein Subjekt muss die verbale Umschreibung, die oben gegeben wurde, nicht notwendigerweise äußern oder denken, um den entsprechenden alief zu haben. Ich gehe davon aus, dass dies ohne weitere Argumentation einleuchtet. Ebenso gilt sowohl für die Intuition als auch für aliefs, dass propositionale Einstellungen auf diesen aufbauen können, die im

288 Die Korrektheit dieser Einordnung des alief als arational hängt von der zugrundeliegenden Rationalitätskonzeption ab. So lassen sich Subjekte für ihre aliefs kritisieren, wie Gendler an anderer Stelle festhält (vgl. Gendler 2010, S. 271). Daher ließen sich aliefs wohl auch zumindest in vielen Fällen wie demjenigen des Skywalk - als irrational beschreiben.

289 Gendler möchte den alief explizit nicht als Akt, sondern als Zustand verstanden wissen, ähnlich zu Aristoteles' Konzept der hexis (vgl. Gendler 2010, S. 263). 
Fall der Intuition als propositionale >Intuitionen, dass $\mathrm{p}<$ (bei gegenläufigen Überzeugungen) bzw. >intuitive Überzeugungen< bezeichnet wurden. Im Fall des alief sind analoge Bezeichnungen plausibel, wie etwa: >alief that ...< und $>$ alief-driven belief that .... ${ }^{290}$

Eine zweite Gemeinsamkeit zwischen alief und Intuition ist, dass beide nicht willentlich erfolgen: Man kann nicht willentlich einen alief haben, sondern dieser stellt sich unweigerlich ein. ${ }^{291}$ Diese Abgrenzung verläuft parallel zu derjenigen, die oben zwischen Intuition und Imagination vorgenommen wurde. Aufgrund deren Realitäts-Insensitivität ist es möglich, Imaginationen willentlich aufzubauen. Demgegenüber sind Intuitionen aufgrund ihrer Realitätssensitivität (auch wenn sie fallibel sind) nicht willentlich herbeiführbar. Auch aliefs sind realitätssensitiv, indem sie Reaktionen auf bestimmte Umweltgegebenheiten darstellen. Wie bei Intuitionen heißt dies auch bei aliefs nicht, dass sie wahr sind. So ist der alief im Skywalk-Beispiel zwar eine Reaktion auf die Realität, aber nicht eine, die einen Sachverhalt korrekt darstellt - daher auch das Spannungsverhältnis zur Überzeugung.

Die dritte Gemeinsamkeit zwischen Intuition und alief ist eine parallel verlaufende Abgrenzung gegenüber der Überzeugung. So ist es gleichermaßen möglich, Überzeugungen entgegenstehende Intuitionen wie auch solche aliefs zu haben. Bezeichnend ist, wie ähnlich sich Beispiele sind, die für die beiden Fälle jeweils naheliegen; etwa diejenigen, die aus den Experimenten des Sozialpsychologen Paul Rozin und Mitarbeitender stammen. ${ }^{292}$ In einem dieser Experimente wurden die Subjekte dazu aufgefordert, Zucker aus einer handelsüblichen Verpackung in zwei Behälter abzufüllen und anschließend selber je ein vorgedrucktes Etikett auf die Behälter zu kleben. Ein Etikett trug die Beschriftung Sodium Cyanide (eine giftige Substanz, worüber die Subjekte informiert waren), das andere die Beschriftung Sugar. Die Resultate zeigten eine signifikant höhere Bereitschaft der Versuchspersonen, eine Lösung aus dem mit Sugar beschrifteten Behälter zu trinken, obwohl ihnen völlig klar sein musste, dass beide Behälter dieselbe Substanz enthielten. Hierbei konnte es sich nur um Zucker und nicht um ein giftiges Pulver handeln. Offenbar befanden sich die Subjekte den beiden Behältern bzw. dem Trinken der Lösungen

290 Gendler unterscheidet zwischen Überzeugungs-entsprechenden vs. -gegenläufigen (belief-concordant vs. -discordant) aliefs (vgl. Gendler 2010, S. 266). Diesen beiden alief-Arten lassen sich die angegebenen propositionalen Einstellungen zuordnen: alief-driven belief für eine auf dem entsprechenden alief aufbauende Überzeugung, alief that für die propositionale Einstellung, welche einem Überzeugungs-gegenläufigen alief entspricht.

291 Vgl. Gendler (2010, S. 271).

292 Vgl. Rozin/Millman/Nemeroff (1986). 
gegenüber in mentalen Zuständen unterschiedlichen Gehalts. Diese Gehalte waren aber gegenläufig zu ihren Überzeugungen. Somit kann der Verweis auf Rozins Experimente zeigen, dass aliefs überzeugungsunabhängig sind, was eine entscheidende Gemeinsamkeit mit Intuitionen ist.

\subsubsection{Unterschiede zwischen Intuition und alief}

Ein erster und entscheidender Unterschied zwischen Intuition und alief ist, dass Intuition eine Ableitung aus mentalen Modellen beinhaltet, während der alief gemäß Gendlers Liste von Merkmalen eine automatische affektive Reaktion auf einen Umweltreiz (oder ein Cluster solcher Reaktionen) ist; jedoch nicht bloß in Form eines Verhaltens, sondern in Form eines Zustandes mit repräsentationalem Gehalt. In Bezug auf das Beispiel des Rozin-Experiments müssen wir uns somit fragen: Kann der »Zyanid-gefährlich«-Gehalt eine automatische affektive Reaktion sein, oder wird dieser Gehalt aus einem mentalen Modell abgeleitet? Nur in letzterem Fall wäre es überhaupt möglich, hier eine Intuition ${ }_{\mathrm{NP}}$ zu verorten und damit gegebenenfalls auch die intuitive Überzeugung der Subjekte zuzulassen (also eine Überzeugung mit den phänomenalen Merkmalen der Unmittelbarkeit und Gewissheit), dass das Zyanid gefährlich ist.

Der Unterschied wird erfassbar, wenn wir die bisherigen Beispiele einander gegenüberstellen:Im Fall des Skywalk haben wir es mit einem Höhenangst-alief zu tun. Das Subjekt zeigt eine automatische und stark affektive Reaktion auf den Umweltreiz der Höhe. Es ist folglich plausibel, dass kein Wissen - wahrscheinlich nicht einmal bestimmte Erfahrungen - diesem alief zugrunde liegt, sondern der alief die direkte und in diesem Sinn »fest verdrahtete« (hard-wired) Reaktion auf den Umweltreiz ist. Im Zyanid-Beispiel hingegen ist es naheliegender, dass eine Ableitung von einem mentalen Modell zugrunde liegt. In einer plausiblen Interpretation dieses Experiments greifen die Versuchspersonen auf bestehendes Wissen zu chemischer Gefahr zurück. ${ }^{293}$ Die Vermutung liegt nahe, dass viele Fälle dem Zyanid-Beispiel ähnlich sind: Eine mit repräsentationalem Gehalt verbundene Reaktion ist in diesen nicht als automatische, direkte Reaktion im Sinne eines alief zu verstehen, sondern als Hervorbringung des Typ 1-Denkens, welches mentale Modelle »abliest«. ${ }^{294}$

293 Damit ist nicht notwendigerweise gemeint, dass sie wissen müssen, dass Zyanid gefährlich ist. Vielmehr wurden auf den Etiketten Gefahrensymbole platziert, sodass ein allgemeines Wissen über chemische Gefahr als Erklärung für die Reaktion der Subjekte ausreicht (vgl. Rozin/Millman/Nemeroff 1986, S. 705).

294 In diesem Sinne interpretiert auch Johnson-Laird vor dem Hintergrund seiner Theorie der mentalen Modelle die Ergebnisse von Rozins Experimenten. Johnson-Laird zufolge 
Eine Gegenprobe kann diese These weiter untermauern: Kann man sich vorstellen, dass ein Subjekt ohne jegliches Wissen über die Gefahren der Höhe im Skywalk-Beispiel Höhenangst zeigt? Vermutlich ist dies zu bejahen. Wie verhält es sich aber mit der Vorstellung der Gefahren in Verbindung mit einer chemischen Substanz? Hier kann man sich kaum vorstellen, dass eine ähnliche Angst- oder Ablehnungsreaktion ebenso voraussetzungslos ist. Vielmehr scheint hier ein Abgleich mit Vorwissen - auch wenn dieser unbewusst geschieht - notwendig. Das Typ 1-Ablesen von mentalen Modellen ist eine plausible Erklärung des Geschehens.

Ein weiterer Unterschied zwischen Intuitionen und aliefs besteht darin, dass auf letzteren keine Überzeugungen aufbauen, welche die phänomenalen Eigenschaften der Intuition (Unmittelbarkeit und Gewissheit) aufweisen. Unmittelbarkeit scheint zunächst auch im Fall eines alief gegeben, da dieser typischerweise sofort auftritt. Doch sollte der Terminus >Unmittelbarkeit< für den Fall vorbehalten bleiben, in dem grundsätzlich auch Mittelbarkeit gegeben sein könnte. Nach diesem Verständnis wäre beispielsweise die Beschreibung »sein Bein schnellte auf den Schlag mit dem Reflexhammer folgend unmittelbar empor« für die entsprechende reflexartige Bewegung als unsinnig zurückzuweisen. Gewissheit lässt sich ebenfalls als phänomenales Merkmal des alief zurückweisen. So wäre unklar, was im Skywalk-Beispiel dem Subjekt als gewiss erscheint. Man stelle sich vor, ein Subjekt wird zu seinen aliefs befragt:Würde es im Skywalk-Beispiel sagen: »Ich weiß auch nicht warum, aber irgendwie bin ich so sicher, dass ich in den Abgrund stürzen werde«? Dies klingt seltsam und wird in der Regel nicht dem entsprechen, wie ein Subjekt seinen alief erlebt. Vielmehr tritt ein alief spontan auf, ohne dass diesem ein epistemischer Status zukommt, weder aus der Außenperspektive noch aus der Perspektive des Subjekts. Gendlers scharfe Abgrenzung des alief vom belief verhindert hier zu Recht den Anschluss an intuitive Überzeugungen.

Ein dritter Unterschied zwischen Intuition und aliefs geht von den alief-Merkmalen >affektiv aufgeladen Intuition kann affektiv aufgeladen sein, wie es anhand der Beispiele aus den Experimenten Rozins deutlich wurde: Hier gehen Intuitionen beispielsweise mit Ekel oder Angst einher. Hingegen ist die affektive Komponente keine notwendige Begleiterscheinung von Intuitionen: Es gibt auch »kalte« Intuitionen. Beispielsweise kann eine mathematische Intuition wie der Spielerfehlschluss in diese Kategorie fallen. Gegenüber dem Merkmal der affektiven Aufladung sind Intuitionen somit gleichermaßen neutral wie etwa Überzeugungen.

machen die Subjekte in diesen Experimenten »emotionale Inferenzen« (vgl. JohnsonLaird 2006, S. 79f.). 
Dagegen ist beim alief Gendlers Zuschreibung der affektiven Aufladung als notwendiges Merkmal plausibel. Dies, da der alief die direkte Reaktion auf Umweltreize darstellt. Als solche gibt er einen starken Impuls zu einer Handlung, indem er beispielsweise vermeidendes Verhalten in Bezug auf einen Reiz auslöst. Zwar kann Handlungsbezogenheit auch im Fall der Intuition gegeben sein, wie am Beispiel der Feuerwehrkommandantin ersichtlich wurde. Aber wie die affektive Aufladung ist auch die Handlungsbezogenheit kein notwendiges Kriterium für eine Intuition. Hier lässt sich etwa das Beispiel der Cartesischen rationalen Intuitionen ins Feld führen: »Ich denke, ich existiere« ist nicht handlungsbezogen, sondern zunächst eine rein kontemplative Einsicht.

Zusammenfassend lässt sich feststellen, dass trotz zunächst stark erscheinender Gemeinsamkeiten die Unterschiede zwischen alief und Intuition beträchtlich sind. Der Vergleich mit dem alief trägt dazu bei, die epistemische Relevanz der Intuition zu unterstreichen, die den entscheidenden Unterschied zu mentalen Zuständen wie dem alief ausmacht.

\section{$5 \cdot 3 \quad$ Intuition und motivierte Irrationalität}

Der Begriff >motivierte Irrationalität hat sich in der philosophischen Diskussion etabliert. ${ }^{295} \mathrm{Er}$ steht für alle Formen irrationalen Denkens und Handelns, deren Irrationalität vom Einfluss motivationaler Faktoren herrührt. In diesem Zusammenhang ist manchmal von »heißer« im Gegensatz zu »kalter« Kognition die Rede. Irrationalität kann auch durch kalte Kognition entstehen, wie die zahlreichen Untersuchungen zu verschiedenen biases und generellen Schwächen des menschlichen Denkens zeigen. ${ }^{296}$ Dass von solcher Irrationalität und somit Fallibilität auch Intuitionen betroffen sein können, stellt den epistemischen Status von Intuitionen nicht grundsätzlich in Frage (siehe Kap. 3.1). Noch nicht behandelt wurde hingegen das Verhältnis der Intuition zu Irrationalität aufgrund von heißer Kognition: Inwiefern ist Intuition auch von motivierter Irrationalität betroffen? Diese Frage ist aus dem folgenden Grund brisant: Stärker noch als Überzeugungen sind Intuitionen in ihrem epistemischen Status durch die mögliche Einflussnahme motivierter Irrationalität in Frage gestellt, da sie aufgrund ihrer starken Verbundenheit

295 Vgl. Pears (1986).

296 Als kalte biases zu bezeichnen sind etwa die verstärkte Reaktion auf lebhaft präsentierte Information sowie der sogenannte confirmation bias, wonach Personen die ihre Hypothese bestätigenden Informationen leichter aufnehmen (vgl. Mele 2004, S. 251f.; Bach 2009, S. 781). 
mit Typ 1-Prozessen der kritischen Evaluation weniger leicht zugänglich sind. Dadurch, dass das Zustandekommen einer Intuition für das erkennende Subjekt im Wesentlichen opak bleibt, ist eine Vielzahl von Intuitionen von der berechtigten Skepsis betroffen, wonach sich in ihnen Wunschdenken oder Selbsttäuschung manifestieren könnte und ihnen aus diesem Grund nicht zu trauen ist. Inwiefern diese Skepsis berechtigt ist, soll im Folgenden untersucht werden.

Die mögliche Nähe von Intuition zu motivierter Irrationalität soll zunächst anhand eines Beispiels veranschaulicht werden. Nehmen wir an, eine blinde psychologische Beraterin habe während eines Gesprächs die intuitive Überzeugung, dass ihr Klient einen Ehering trägt, wobei keine direkten Hinweise diesbezüglich aus dem Gespräch folgen. ${ }^{297}$ Die intuitive Überzeugung stellt sich im Nachhinein als falsch heraus. In Wahrheit war ein bestimmter Wunsch der Beraterin für ihre Überzeugung verantwortlich, dass der Klient einen Ehering trägt. ${ }^{298}$ Beispielsweise könnte sie das Ideal verinnerlicht haben, Männer mittleren Alters sollten verheiratet sein. Da sie aufgrund der Stimme und der Erzählungen ihren Klienten als Mann mittleren Alters einstuft, wird ihr Wunsch wirksam. ${ }^{299}$ Sie kommt dadurch zu einer als Wunschdenken einzuordnenden Überzeugung: Weil die Beraterin sich wünscht, ihr Klient möge einen Ehering tragen, bildet sie die Überzeugung aus, dass ihr Klient einen Ehering trägt. Die Irrationalität dieser Überzeugung zeigt sich auch darin, dass sie sich über deren motivationale Konstitution keine Rechenschaft gibt, sondern diese als wunschunabhängig einordnet - im vorliegenden Fall dadurch, dass sie den Gehalt der Überzeugung als intuitiv, d.h. mit ihren diagnostischen Fähigkeiten verbunden, einordnet. Diese falsche Einordnung wird dadurch begünstigt, dass eine Intuition durch nicht direkt zugängliches Typ 1-Schließen zustande kommt; denn auf der Typ 1-Ebene spielt sich auch die Einflussnahme der Motivation auf den repräsentierten Gehalt ab. Dadurch schleicht sich - so

297 Dieses Beispiel ist einer realen Diskussion unter Beraterinnen auf dem Weblog der alternativen Therapeutin Anna Sayce entnommen (vgl. Sayce o. J.).

298 Überzeugungen können in Graden auftreten (vgl. Schwitzgebel 2015). Auch wenn eine gewisse Unsicherheit der Therapeutin bezüglich der Ehering-Proposition zurückbleibt, ist dadurch nicht in Abrede gestellt, dass es sich um eine Überzeugung handelt. So stellt sich die Frage, ob diese Überzeugung eine intuitive (wie im Rahmen dieser Arbeit charakterisiert) oder eine durch motivationale Faktoren konstituierte und dadurch irrationale ist.

299 Dieser Wunsch kann der Beraterin bewusst zugänglich sein oder unbewusst bleiben. Somit ist keine propositionale Repräsentation des Wunsches ( $>S$ wünscht, dass $\mathrm{p}<$ ) notwendig, jedoch möglich. In jedem Fall hat die Beraterin im Beispiel keinen Zugriff auf die Art und das Maß, in dem ihr Wunsch sich auf die Überzeugung auswirkt, dass ihr Klient einen Ehering trägt. 
die naheliegende Vermutung - verzerrende Motivation unmerklicher als im Fall der Wahrnehmungs- oder der deduktiv hergeleiteten Überzeugung in den Prozess der Überzeugungsbildung ein.

Trifft diese Analyse zu, kann das Beispiel veranschaulichen, wie nahe sich Intuition und Ausprägungen motivierter Irrationalität kommen können. Dadurch stellt sich die Aufgabe, das Verhältnis zwischen motivierter Irrationalität und Intuition genauer zu betrachten. Das Ziel ist dabei, den epistemischen Status der Intuition gegenüber Skeptikern zu verteidigen, die auf die unkontrollierbare Einflussnahme motivierter Irrationalität hinweisen. Die Diskussion soll auf Wunschdenken (wishful thinking) sowie auf Selbsttäuschung (self-deception) beschränkt werden, die beiden gegenwärtig stark diskutierten Formen der motivierten Irrationalität. ${ }^{300}$

\subsubsection{Meles Verständnis der motivierten Irrationalität}

In klassischen Erklärungsansätzen der Selbsttäuschung wurde diese auf der Folie der interpersonalen Täuschung konstruiert. Demnach täuscht ein Subjekt sich selbst, wie es jemanden anderes täuschen kann. ${ }^{301}$ Diese Ansätze sind der Problematik ausgeliefert, dass Subjekten die Absicht sich selbst zu täuschen zugeschrieben werden muss. Dies erzeugt Mele zufolge zwei Paradoxa, die er das statische und das dynamische Paradox nennt. Dem statischen Paradox zufolge muss der Intentionalismus dem Subjekt sowohl die durch die Selbsttäuschung konstituierte falsche Überzeugung als auch die wahre Überzeugung zugleich zuschreiben, denn nur so ist denkbar, dass das Subjekt sich selbst täuscht. Die Zuschreibung zweier sich widersprechender Überzeugungen scheint aber problematisch. Ein weiteres Problem ergibt sich durch das dynamische Paradox: Dem Subjekt muss die Intention zugeschrieben werden, sich selbst zu täuschen. Da Intentionen üblicherweise als bewusst gelten, fragt sich, wie es dem Subjekt gelingen kann, sich zu täuschen, insofern ihm die Täuschungsintention bewusst ist. ${ }^{302}$ Aufgrund dieser Schwierigkeiten lehnt Mele den Intentionalismus in Bezug auf Selbsttäuschung ab und stellt ihm einen Minimalismus gegenüber:

300 Es ist plausibel, dass Selbsttäuschung auch im Bereich der kalten Kognition, d.h. unmotiviert, auftreten kann. So kann Selbsttäuschung auch als mangelhafte Sicht auf die eigenen psychischen Zustände, insbesondere Handlungsgründe verstanden werden (vgl. Patten 2003). Wenn im Folgenden von Selbsttäuschung die Rede ist, ist immer die Selbsttäuschung als Form motivierter Irrationalität gemeint.

301 Vgl. etwa Demos (1960), Pears (1986), McLaughlin (1988), Davidson (2004).

302 Vgl. Mele (1997, S. 92). 
I suggest that the following conditions are jointly sufficient for entering selfdeception in acquiring a belief that $p$.

1. The belief that $p$ which $S$ acquires is false.

2. $S$ treats data relevant, or at least seemingly relevant, to the truth value of $p$ in a motivationally biased way.

3. This biased treatment is a nondeviant cause of $S^{\prime}$ acquiring the belief that $p$.

4. The body of data possessed by $S$ at the time provides greater warrant for $\sim p$ than for $p .^{303}$

Im minimalistischen Erklärungsmodell der Selbsttäuschung wird also auf eine Selbsttäuschungs-Absicht des Subjekts verzichtet. Damit distanziert sich diese Erklärung von der Herkunft des Wortes: Selbsttäuschung kann nicht mehr auf der Folie interpersonaler Täuschung verstanden werden. Jedoch sollte der Wortherkunft in diesem Fall keine Autorität eingeräumt werden. Entscheidend ist, dass der Erklärungsansatz ein im Alltagsverständnis bekanntes und oft als >Selbsttäuschungく bezeichnetes Phänomen erfassen kann. Um dies zu bestätigen, empfiehlt sich der Blick auf paradigmatische Beispiele, wie die bereits angeführten: Die erwähnten Elemente des minimalistischen Modells lassen sich mühelos auf diese anwenden. ${ }^{304}$

Neben den Stärken des minimalistischen Modells scheint jedoch die erste der von Mele genannten Bedingungen verzichtbar oder gar problematisch zu sein: Die Selbsttäuschungs-Überzeugung muss nicht zwingend falsch sein. Um dies zu erkennen, lassen sich etwa Beispiele konstruieren, in denen das Subjekt zwar eine wahre Überzeugung erwirbt, dieser Erwerb aber nicht aufgrund der Evidenz, sondern aufgrund der Motivation erfolgt. So könnte es im Beispiel der Beraterin tatsächlich der Fall sein, dass der Klient einen Ehering trägt. Wenn die Beraterin allerdings ohne Evidenz oder gar trotz überwiegend dagegensprechender Evidenz (aus dem Gespräch mit dem Klienten) zu dieser korrekten intuitiven Überzeugung gelangt, hat sie die Wahrheit mehr oder weniger zufällig getroffen. Es wäre nicht folgerichtig, aufgrund dieses Zufallstreffers - während alle anderen Bedingungen der Selbsttäuschung gegeben sind - den Fall nicht mehr als Selbsttäuschung einzuordnen. Aufgrund dieser Überlegungen werde ich im Folgenden weiter für den Minimalismus

303 Mele (1997, S. 95).

304 Mele führt weitere aufschlussreiche Beispiele an, wie z.B. die Folgenden:

Seemingly, some parents who are self-deceived in believing that their children have never experimented with drugs and some people who are self-deceived in believing that their spouses have not had affairs have never believed that these things have happened. Owing to self-deception, they have not come to believe the truth, and perhaps they never will.

(Mele 2004, S. 249) 
argumentieren und diesen in Bezug auf die Erklärung motivierter Kognition im Bereich der Intuition anwenden, jedoch ohne die problematische erste Bedingung Meles zu übernehmen. Dies zieht eine Änderung der vierten Bedingung nach sich: Die Evidenz, über die S zu einem gegebenen Zeitpunkt verfügt, könnte zufällig in Einklang mit der Selbsttäuschung stehen. Entscheidend ist, dass $\mathrm{S}$ die entsprechende Überzeugung nicht aufgrund dieser Evidenz ausbildet.

Der minimalistische Ansatz wurde bisher nur in Bezug auf Selbsttäuschung ausgeführt. Dem Minimalismus folgend besteht zwischen Wunschdenken und Selbsttäuschung aber kein grundsätzlicher Unterschied. Dies rührt daher, dass die Intention, die ein entsprechendes Unterscheidungskriterium zwischen den beiden Arten motivierter Irrationalität lieferte, wegfällt. So lässt sich die Unterscheidung zwischen einem auf den relevanten Gehalt zielenden Wunsch (zu Wunschdenken führend) und einem anderen, für den Gehalt relevanten aber nicht auf diesen abzielenden Wunsch (zu Selbsttäuschung führend) nicht mehr treffen. Dementsprechend sieht Mele zwischen Wunschdenken und Selbsttäuschung keinen grundlegenden Unterschied, sondern einen graduellen Übergang, wobei in beiden Fällen die motivationale Einflussnahme den mangelhaften Realitätsbezug des Gehaltes erklärt. ${ }^{305} \mathrm{Ob}$ es sich bei einem einzelnen Vorkommnis jeweils eher um Wunschdenken oder um Selbsttäuschung handelt, wird dem Minimalismus zufolge durch die Stärke der gegen den motivierten Gehalt sprechenden Evidenz bestimmt. In paradigmatischen Fällen der Selbsttäuschung hält das Subjekt diese gegen eine stark für das Gegenteil sprechende Evidenz aufrecht, während beim Wunschdenken keine solche Gegenevidenz besteht.

\subsubsection{Intuitionen des Wunschdenkens und der Selbsttäuschung}

Mele beschäftigt sich in seinem Erklärungsansatz motivierter Irrationalität nicht mit Intuition. Im Folgenden soll daher aufgezeigt werden, inwiefern dieser Ansatz auch auf Intuition angewandt werden kann. Dies wird eine Erklärung für den einleitend dargestellten Befund liefern, wonach Intuitionen und Fälle motivierter Irrationalität einander nahekommen können.

Der wesentliche Schritt, um Meles Ansatz auf die Intuition anwenden zu können, besteht darin, dessen Fixierung auf Überzeugungen aufzuheben. Eine Vorbereitung dazu ist bereits mit der obigen Zurückweisung von Meles erstem Kriterium erfolgt, wonach S eine falsche Überzeugung erwirbt. Das Argument hat aber noch nicht in Frage gestellt, dass $S$ überhaupt eine Überzeugung erwirbt, deren Gehalt durch motivierte Irrationalität bestimmt ist. Dieser Schritt

305 Vgl. Mele (1997, S. 100). 
soll nun getan werden. Ohne Weiteres kann auch eine Intuition Trägerin der Selbsttäuschung bzw. des Wunschdenkens sein, der keine Überzeugung entspricht. Um dies zu erkennen, können wir das Beispiel der Beraterin leicht modifizieren: Nehmen wir an, die blinde Beraterin habe durch einen Hinweis im Gespräch starke Evidenz dafür, dass der Klient wahrscheinlich keinen Ehering trägt (beispielsweise erzählt er von einer kürzlichen Scheidung). Dennoch kann sie sich der Intuition nicht erwehren, wonach genau dies der Fall ist. Sie weist aber die Intuition für sich zurück, da sie sich nur an klarer Evidenz orientieren will. Dieser plausible Fall ist parallel zu demjenigen des Mathematikers konstruiert, der sich der Spielerfehlschluss-Intuition nicht erwehren kann, ihren Gehalt aber keinesfalls akzeptiert.

Intuitionen der Selbsttäuschung und des Wunschdenkens sind keine Überzeugungen. Wie ersichtlich wurde, lässt sich Subjekten eine Intuition zuschreiben, die nicht mit einer entsprechenden Überzeugung einhergeht. Doch auch in diesem Fall weist die Intuition das phänomenale Merkmal der Gewissheit auf, denn es handelt sich bereits um eine >Intuition, dass $\mathrm{p}<$, nicht mehr um eine Intuition $\mathrm{NP}_{\mathrm{NP}}$. Dadurch kann der minimalistische Ansatz sinnvoll auf die Intuition angewandt werden, ohne die Konzepte der Selbsttäuschung und des Wunschdenkens ins Beliebige ausufern zu lassen. Der entscheidende Punkt der motivierten Irrationalität, die subliminale Wirkung der Motivation auf realitätssensitive mentale Zustände, bleibt in einem auf die Intuition bezogenen minimalistischen Ansatz bestehen: Die Motivation zeigt sich nicht direkt, sondern nimmt auf der Ebene der Typ 1-Kognition Einfluss auf die Prozesse der Intuitions- und Überzeugungsbildung. Daraus ergeben sich die folgenden Beschreibungen der motivierten Irrationalität in Bezug auf Intuition:

\section{Motivierte Irrationalität: Selbsttäuschung}

Die folgenden Bedingungen sind gemeinsam hinreichend dafür, dass $\mathrm{S}$ einer Selbsttäuschung in Form einer Intuition (intuitive Überzeugung oder >Intuition, dass $\mathrm{p}<$ ) unterliegt: ${ }^{306}$

(1) S behandelt relevante oder relevant scheinende Evidenz für die intuitive Überzeugung oder >Intuition, dass $\mathrm{p}<$ auf eine motivational beeinflusste Weise.

(2)Diese motivational beeinflusste Behandlung ist eine direkte Ursache für S' Erwerb der intuitiven Überzeugung oder der >Intuition, dass $\mathrm{p}<{ }^{307}$

306 Meles erste Bedingung wird nun weggelassen, da sie die Selbsttäuschung als Überzeugung fixierte.

307 Hier übersetze ich den von Mele verwendeten Ausdruck nondeviant cause vereinfachend mit >direkte Ursacheく. Für eine präzise Darstellung von Devianz bei kausalen Verknüpfungen siehe Shope (1999, S. 127-136). 
(3) S ignoriert bei der Bildung der intuitiven Überzeugung oder >Intuition, dass $\mathrm{p}$ < Teile der relevanten und für $\mathrm{S}$ zum gegebenen Zeitpunkt verfügbaren Evidenz.

\section{Motivierte Irrationalität: Wunschdenken}

Die folgenden Bedingungen sind gemeinsam hinreichend dafür, dass S einem Wunschdenken in Form einer intuitiven Überzeugung oder >Intuition, dass $\mathrm{p}<$ unterliegt:

(1) S behandelt relevante oder relevant scheinende Evidenz für die intuitive Überzeugung oder die >Intuition, dass $\mathrm{p}<$ auf eine motivational beeinflusste Weise.

(2) Diese motivational beeinflusste Behandlung ist eine direkte Ursache für S' Erwerb der intuitiven Überzeugung oder >Intuition, dass p<.

Die von Mele nur für die Selbsttäuschung aufgeführten Bedingungen wurden damit auf das Wunschdenken übertragen. Dabei wurde die dritte Bedingung ganz weggelassen, da im Fall des Wunschdenkens keine Evidenz ignoriert wird, sondern das Wunschdenken die Lücke nicht vorhandener Evidenz füllt. Dies berücksichtigt die verbreitete Auffassung, wonach Wunschdenken eine schwächere Form der Irrationalität ist als Selbsttäuschung. ${ }^{308}$

Zusammenfassend lässt sich eine hohe Passung zwischen dem Minimalismus in der Theorie motivierter Irrationalität (bei entsprechenden Modifikationen) und der hier vertretenen Intuitionskonzeption feststellen. Indem der Minimalismus auf intentionalistische Erklärungen der motivierten Kognition verzichtet, ermöglicht er anzuerkennen, dass Motivation im Typ 1-Denken, also subliminal, auf die Erkenntnis Einfluss nimmt. Somit lässt sich der Minimalismus sinnvoll auf die Intuition anwenden, ohne dadurch in eine Beliebigkeit der Konzepte >Selbsttäuschung $<$ und >Wunschdenken $<$ abzugleiten.

\subsubsection{Unterscheidung motivierte vs. biased Intuition}

Eine andere Abgrenzungsfrage in Bezug auf das Verhältnis zwischen Intuition und motivierter Kognition ist noch offen: Gibt es einen Unterschied zwischen einer fehlerhaften Intuition aufgrund eines kognitiven biases und einer fehlerhaften Intuition aufgrund motivierter Irrationalität? Zunächst liegt mit Verweis auf Beispiele wie den Spielerfehlschluss auf der Hand, dass Irrationalität auch auftreten kann, ohne motiviert zu sein. ${ }^{309}$ Doch wie verhält es sich beispielsweise mit dem Bestätigungs-bias oder mit diskriminierenden biases in Bezug auf Aussehen, Alter und Geschlecht? Hier ist die Abgrenzung $\mathrm{zu}$ motivierter Irrationalität schwieriger. Um diese Abgrenzung dennoch

308 Für eine genauere Behandlung des Wunschdenkens siehe Maller (2013).

309 Vgl. Bach (2009, S. 781). 
vornehmen zu können, kann auf den »motivationalen Haushalt« des jeweiligen Subjekts Bezug genommen werden. Lässt sich durch eine Veränderung dieses motivationalen Haushalts die fehlerhafte Intuition potentiell korrigieren oder ist dies notwendigerweise unmöglich? Im ersten Fall ist motivierte Irrationalität für den Fehler verantwortlich, im zweiten handelt es sich um einen bias und somit um einen aus externer und objektiver Perspektive erklärbaren Fehler der Intuition. ${ }^{310}$ Auf die Beispiele des Bestätigungs-bias und der diskriminierenden biases zurückzukommend lässt sich der Unterschied im Folgenden veranschaulichen.

Subjekte werden in der Regel vorrangig nach Evidenz suchen, die ihre Hypothese bestätigt und solche eher vernachlässigen, die sie widerlegt. Es ließe sich experimentell überprüfen, ob dies bei einem gegebenen Subjekt selbst dann der Fall ist, wenn weder ein auf den Gehalt der Hypothese bezogener Wunsch vorliegt, noch ein anderer Wunsch mit dem Gehalt der Hypothese ursächlich zusammenhängt. Experimente zum Bestätigungs-bias wurden mit einer Vielzahl von Subjekten durchgeführt. ${ }^{311}$ Die Tatsache, dass sich der bias bei allen oder zumindest der Mehrzahl der Subjekte zeigt, lässt darauf schließen, dass der bias unabhängig von einer subjektiven Motivation besteht, da Subjekte motivational ganz unterschiedlich konstituiert sind. Dasselbe lässt sich in Bezug auf diskriminierende biases sagen, die beispielsweise in Experimenten mit fingierten Stellenbewerbungen festgestellt wurden: Bewerbende mit Namen ausländischer Herkunft wurden signifikant weniger häufig zum Bewerbungsgespräch eingeladen. ${ }^{312}$ Viele dieser Subjekte hegen keine Wünsche bezüglich Rassendiskriminierung, trotzdem fallen sie den biases anheim. Es entbehrt einer Grundlage, all diesen Subjekten verborgene rassistische Motivationen zu unterstellen.

An dieser Stelle muss das Verhältnis zwischen unmotivierten und motivierten falschen Intuitionen jedoch differenzierter betrachtet werden. So ist unter Psychologinnen und Psychologen die Auffassung durchaus verbreitet, wonach sich die beiden letztgenannten Beispiele für intuitive

310 Um zu betonen, wie wichtig diese Erklärbarkeit ist, sei folgender Punkt in Erinnerung gerufen: Die Erklärung falscher Intuitionen bedeutet im Wesentlichen, dass diese in ein größeres Bild eingeordnet werden können, in dem sich Intuition als grundsätzlich epistemisch wertvoll erweist (vgl. Kap. 4.2). D.h., die falschen Intuitionen aufgrund kalter Kognition müssen sich plausibel als aus epistemologischer Sicht in Kauf zu nehmende Nebenwirkungen einer grundsätzlich hochzuschätzenden Erkenntnisquelle einordnen lassen. Dieses Erfordernis besteht bei falschen Intuitionen aufgrund motivierter Irrationalität nicht.

311 Vgl. Baron (2000, S. 203f.).

312 Vgl. Bertrand/Mullainathan (2004). 
biases auf die Motivation zurückführen lassen. So wurde etwa im Fall des Bestätigungs-bias der Versuch unternommen, diesen auf Wunschdenken zurückzuführen, oder auf den Wunsch, den teuersten Fehler zu vermeiden. ${ }^{313}$ Dass die Genese intuitiver biases also - auf Ebene des Subjekts oder in der evolutionären Entwicklung - mit Motivation ursächlich verknüpft ist, sowie auch, dass Motivation eine wichtige Rolle in der jeweiligen Aufrechterhaltung des bias spielen kann, muss auch im Fall der primär kalten Irrationalität als Möglichkeit stets angenommen werden. Es ist letztlich von einem Kontinuum zwischen gänzlich motivierten und gänzlich unmotivierten Intuitionen auszugehen. ${ }^{314}$ Das Bestehen eines Kontinuums stellt die Unterscheidung der beiden Pole jedoch nicht infrage. Je mehr die relevante Intuition auf überindividuellen motivationalen Faktoren beruht oder gar ausschließlich durch kalte Kognition erklärbar ist, desto leichter kann eine allgemeine und externalistische Erklärung ihrer Zuverlässigkeit oder Fallibilität, beispielsweise via Adaptations-Hypothese, gegeben werden. Dies muss aber eine empirische Zielsetzung sein, die hier nicht weiterverfolgt werden kann.

Als Fazit zum Verhältnis zwischen Intuition und motivierter Irrationalität ergibt sich, dass Intuition als realitätssensitive Erkenntnisgattung den verfälschenden Einflüssen motivationaler Faktoren ebenso ausgesetzt sein kann wie etwa die deduktiv erlangten Überzeugungen. Doch wie wir Überzeugungen deswegen nicht ihre epistemische Bedeutung absprechen (z.B. als Träger von Gründen), sollten wir dies auch bei Intuitionen nicht tun.

Dennoch zeigt sich hier, dass sich eine Klasse von Intuitionen als epistemisch besonders wertvoll erweist: die selbstevidenten Intuitionen. Nachdem hier bisher für eine allgemeine und psychologisch fundierte Intuitionskonzeption plädiert wurde und die selbstevidenten Intuitionen dadurch ihren Sonderstatus verloren, dürfen sie nun wieder in besonderem Licht erscheinen. Zeigt sich nämlich in einer Intuition deren Wahrheit selbstevident an, entfallen alle sorgenvollen Überprüfungen hinsichtlich möglicher Verzerrungen durch motivierte Irrationalität. Beispielsweise lässt sich unter der Annahme, dass Descartes' intuitiver Schluss von >ich denke< auf >ich existiere< statthaft und selbstevident ist, jede Frage nach möglichen Verzerrungen dieser Intuition durch motivationale Faktoren als überflüssig zurückweisen. Die Wahrheit dieser Intuition zeigt sich schlicht in der Intuition selbst. Dies soll aber nicht zu einer Wiedereingrenzung des Intuitionsbegriffs auf selbstevidente Intuitionen

313 Vgl. Nickerson (1998, S. 197); Mele (1999, S. 120-124).

314 Verwandt mit der Kontinuums-Hypothese ist diejenige, wonach Elemente der kalten Kognition als Mediatoren für Effekte der motivierten Kognition auftreten können und vice versa (vgl. Nickerson 1998, S. 198). 
führen. Sinnvoller ist es, die Beschreibung der Intuition als Erkenntnisgattung so weit zu lassen, wie es ihre allgemeinen Merkmale vorgegeben haben, ohne dabei in Abrede zu stellen, dass bestimmte ihrer Ausprägungen - hier die selbstevidenten Intuitionen - einen besonderen epistemischen Status genießen.

\subsection{Intuition und Delusion}

Die nun folgende Untersuchung zum Verhältnis zwischen Intuition und Delusion weist Ähnlichkeiten mit derjenigen des vorigen Kapitels zur motivierten Irrationalität auf. So geht es auch hier um den Einfluss irrationaler Faktoren auf die Bildung einer Intuition. ${ }^{315}$ Zunächst sollen Grundlagen der Delusion geklärt werden, um anschließend das Verhältnis zwischen Intuition und Delusion bestimmen zu können.

\subsubsection{Psychologische Verständnisse der Delusion}

Als Ausgangspunkt der Diskussionen um Delusion dient oftmals der entsprechende Eintrag im Diagnostic and Statistical Manual of Mental Disorders (DSM-V):

Delusions are fixed beliefs that are not amenable to change in light of conflicting evidence. Their content may include a variety of themes (e.g. persecutory, referential, somatic, religious, grandiose). [...] Delusions are deemed bizarre if they are clearly implausible and not understandable to same-culture peers and do not derive from ordinary life experiences. [...] The distinction between a delusion and a strongly held idea is sometimes difficult to make and depends in part on the degree of conviction with which the belief is held despite clear or reasonable contradictory evidence regarding its veracity. ${ }^{316}$

315 Inwieweit Delusion selbst als Fall der motivierten Irrationalität gilt, bzw. sich zumindest mit diesem Bereich überschneidet, ist umstritten. Hier soll der Fokus auf der Intuition bleiben, weshalb die Frage nach dem Verhältnis zwischen Delusion und motivierter Irrationalität nicht weiter behandelt wird. Zu dieser Frage siehe u.a. Mele (2008), Bortolotti (2010, S. 47-41), Bayne/Fernandez (2008, S. 2f.).

316 American Psychiatric Association (2013, S. 86) - Die Definition wurde gegenüber der vorherigen Ausgabe (DSM-IV) verändert, dies aufgrund verschiedener Einwände und Gegenbeispiele, die in der philosophisch-psychologischen Diskussion vorgebracht worden waren. Beispielsweise werden Delusionen nicht länger als zwingend falsch beschrieben, ihr Gehalt kann auch auf das Subjekt selbst, statt bloß auf externe Realität bezogen sein und ein Kontinuum zwischen Delusionen und anderen fixen Ideen mit entsprechenden Gemeinsamkeiten wird anerkannt (vgl. Bortolotti 2013). 
Delusionen sind ein Symptom unterschiedlicher psychischer Krankheiten, wie beispielsweise der Schizophrenie oder der Demenz. Zur Veranschaulichung seien drei bekannte Delusionen genannt: Bei der Capgras-Delusion sind die betroffenen Subjekte davon überzeugt, dass ihre Partnerin bzw. ihr Partner durch einen Doppelgänger ersetzt wurde. Die Fregoli-Delusion besteht in der Überzeugung, man werde von bekannten, jedoch aufgrund ihrer Verkleidung nicht erkennbaren Personen verfolgt. Unter der Cotard-Delusion leidende Subjekte sind der Überzeugung, sie seien tot. ${ }^{317}$

Da es keinen Konsens über die Ursachen und die Entstehung der Delusionen gibt, fokussieren Definitionen wie die obige aus dem DSM-V auf beobachtbare Eigenschaften. ${ }^{318}$ Bemerkenswert ist, dass Delusionen im DSM-V, wie auch in der übrigen Forschungsliteratur verbreitet, als Überzeugungen bezeichnet werden. Entscheidend ist dabei eine hohe Resistenz dieser Überzeugungen gegenüber für das Subjekt eigentlich vorhandener Evidenz, die gegen sie spricht. Ebenfalls im DSM-V genannt wird eine bedeutende Abweichung der Überzeugungen von epistemischen Normen der umgebenden Kultur. Diese Elemente (Überzeugung, gegen Evidenz stehend, von umgebenden epistemischen Normen deutlich abweichend) lassen sich gemeinsam als epistemische Merkmale der Delusion bezeichnen. Die DSM-V-Definition fokussiert auf die erwähnten Merkmale, weist aber darauf hin, dass dadurch Abgrenzungsschwierigkeiten zu anderen »fixen Ideen« bestehen, die kaum als Delusionen gelten dürften. Insbesondere aus diesem Grund sollte als weiteres notwendiges Merkmal einer Delusion eine negative bis destruktive Auswirkung auf das Subjekt und/oder dessen nähere Umgebung aufgenommen werden. ${ }^{319}$

Grundlegend lässt sich zwischen >1-Faktor<- und >2-Faktoren<-Erklärungsansätzen zur Delusion unterscheiden, wobei sich >Faktor $1<$ auf die zugrundeliegende Erfahrung, >Faktor $2 \ll$ auf die nachgelagerten inferentiellen Prozesse bezieht. Die 1-Faktor-Erklärung wird von Brendan Maher vertreten. Maher zufolge entstehen delusionale Überzeugungen, ebenso wie normale Überzeugungen, aus dem Versuch heraus, pathologische Wahrnehmungen zu erklären. ${ }^{320}$ Es sind demnach die Wahrnehmungen, die defektiv sind, während die inferentiellen Prozesse der betroffenen Subjekte sich nicht von denjenigen anderer Subjekte unterscheiden. ${ }^{321}$ Einer gleichen Wahrnehmungsanomalie

317 Vgl. u.a. Coltheart (2005, S. 72).

318 Vgl. Bortolotti (2010, S. 22).

319 Vgl. Bortolotti (2010, S. 24).

320 Vgl. Maher (1999, S. 15of.).

321 Maher führt verschiedene Pathologien der Wahrnehmung auf, die - aufgrund folgender und in sich rationaler Inferenzen - zu Delusionen führen. Im Rückgriff auf Jaspers' 
ausgesetzt könnte jedes beliebige Subjekt zu einer Delusion kommen, ohne dass weitere Probleme in Bezug auf die inferentiellen Fähigkeiten des Subjekts vorliegen. Die Delusion wird in der 1-Faktor-Erklärung entsprechend als an sich rationale Strategie zur Erklärung von Wahrnehmungsanomalien verstanden.

Max Coltheart und Mitarbeitende kritisieren diese Erklärung der Delusion. Unter Berufung auf eine Vielzahl empirischer Befunde zeigen sie auf, dass Erfahrungsanomalien keine hinreichende Bedingung für eine Delusion darstellen. ${ }^{322}$ Dies lässt sich etwa am Beispiel der Capgras-Delusion zeigen. Zunächst ließ sich feststellen, dass eine Wahrnehmungsanomalie am Ursprung der Delusion liegt; hier die fehlende Reaktion des limbischen Systems auf das bekannte Gesicht, was dazu führt, dass das Gefühl der Vertrautheit beim Anblick des Gesichts des Partners ausbleibt (= Faktor 1). Jedoch wurde nachgewiesen, dass Subjekte diese Wahrnehmungsanomalie aufweisen können ohne die Capgras-Delusion zu entwickeln. Daher wird ein zweiter Faktor angenommen, der zur Delusion führt. Dieses zweite Defizit führt dazu, dass die aufgrund des ersten Defizits zustande gekommene Überzeugung nicht als unplausibel zurückgewiesen, sondern aufrechterhalten wird. ${ }^{323}$ Kern des zweiten Defizits ist, dass relevante Evidenz gegen die problematische Überzeugung nicht adäquat aufgenommen wird. Empirische Befunde deuten darauf hin, dass dieses zweite Defizit aufgrund von Beschädigungen des rechten Frontallappens auftreten kann. Solche Beschädigungen sind im Fall der Patienten, die zwar die anomale Wahrnehmung aufweisen, aber aufgrund weiterer Evidenz von der delusionalen Überzeugung Abstand nehmen, nicht gegeben. ${ }^{324}$

\subsubsection{Die Möglichkeit delusionaler Intuitionen}

Warum es von Interesse ist, das Verhältnis zwischen Intuition und Delusion zu untersuchen, lässt sich wiederum mit Verweis auf Beispiele zeigen. So lässt sich das im vorigen Kapitel ausgeführte Beispiel der Beraterin in diese Richtung modifizieren: Lassen wir die Überzeugung der Beraterin nur etwas abstruser werden und schließen auch eine negative Auswirkung auf das

Unterscheidung zwischen primären und sekundären Delusionen bezieht sich Maher auf ein klassisches Beispiel für erstere: Einem Mann, der in einem Café die Marmortische betrachtete, schien unmittelbar gewiss, dass diese Tische auf das nahende Ende der Welt hindeuten. In Mahers Ansatz wird dies als eine Delusion aufgrund einer autonomen Aktivierung von Signifikanzgefühlen beschrieben. Die Delusion ist der in sich vernünftige Ansatz, mit dem sich das Subjekt dieses pathologische Signifikanzgefühl beim Anblick der Marmortische erklärt (vgl. Maher 1999, S. 559; siehe auch Davies 2008, S. 72f.).

322 Vgl. u.a. Coltheart/Menzies/Sutton (2010, S. 266-269).

323 Vgl. Coltheart (2005, S. 73).

324 Vgl. Coltheart (2007, S. 1044-1052f.). 
Umfeld mit ein, liegt die Zuschreibung einer Delusion nahe. Beispielsweise könnte die Beraterin - ohne intersubjektiv gegebene Evidenz - zur intuitiven Überzeugung gelangen, ihr Klient werde morgen einen Massenmord begehen. Eine solche intuitive Überzeugung wird zumindest der Beraterin selbst schaden, vermutlich in Form tiefer Verstörung, womöglich aber auch dem Klienten und dem weiteren Umfeld, abhängig davon, welche Handlungen die Beraterin aufgrund dieser intuitiven Überzeugung ausführen zu müssen meint. Wann schlägt eine Intuition in eine Delusion um? Im Folgenden lässt sich bestätigen, dass gewisse Intuitionen auch Delusionen sein können. Doch die Untersuchung zu möglichen Zusammenhängen zwischen Intuition und Delusion kann zugleich aufzeigen, dass daraus keine neue Bedrohung des epistemischen Status der Intuitionen erwächst.

Grundsätzlich bestehen vier Möglichkeiten, in welchem Verhältnis Intuitionen zu Delusionen stehen können, wenn sie - was hier angenommen werden soll - überhaupt etwas miteinander zu tun haben. Erstens können Delusionen eine Subklasse von Intuitionen sein, zweitens kann sich die Menge der Delusionen mit derjenigen der Intuitionen überschneiden, drittens können Intuitionen koextensiv mit Delusionen oder viertens eine Subklasse der Delusionen sein. ${ }^{325}$ Da die dritte und vierte Möglichkeit implizieren, dass alle Intuitionen Delusionen sind, können sie sogleich als überzogen zurückgewiesen werden. Es dürfte allgemein akzeptiert sein, dass viele Intuitionen nicht entgegen jeglicher Evidenz vorliegen, sondern - ganz im Gegenteil - gar selbstevident sein können. Auch bei nicht zur Evidenz passenden Intuitionen ist in vielen Fällen das Kriterium der Destruktivität nicht gegeben. Mit Sicherheit sind also nicht alle Intuitionen Delusionen. Die erste und die zweite Möglichkeit sind hingegen genauer zu prüfen.

Mit einem Intuitionsverständnis, in dessen Zentrum das nichtbegriffliche Schließen ausgehend von mentalen Modellen steht, kann die Nähe zur Delusion aufgrund von Colthearts 2-Faktoren-Ansatz plausibel erklärt werden. Faktor 1, die pathologische Wahrnehmung, lässt sich mit dem mentalen Modell gleichsetzen, von dem eine Intuition ausgeht. Tatsächlich scheint die Pathologie in den meisten Fällen nicht einen einzelnen Wahrnehmungsgehalt, sondern ein ganzes mentales Modell zu betreffen. Das fehlende Gefühl der Vertrautheit beim Anblick des Partners illustriert dies gut: Der Anblick des Partners entspricht nicht einer isolierten Wahrnehmung. Vielmehr sind

325 Mit >Intuitionen können eine Subklasse der Delusionen sein< ist hier präziser gemeint: Die Menge der mentalen Zustände, auf welche aufgrund ihrer Eigenschaften die Bezeichnung >Intuition zutrifft, ist in der Menge der mentalen Zustände, auf welche aufgrund ihrer Eigenschaften die Bezeichnung >Delusion` zutrifft enthalten. 
seine Gesichtszüge, Statur, Haarfarbe, sowie im weiteren Sinn von >Anblick auch Gestik, Mimik, Stimme, usw. darin enthalten. Die fehlende Vertrautheit bezieht sich auf ein Ensemble solcher Elemente, also auf ein mentales Modell. Es handelt sich um ein defizitäres mentales Modell, insofern es nicht eine korrekte Repräsentation der Wirklichkeit ist, da zu einer korrekten Repräsentation eines einem nahestehenden Menschen auch das Gefühl der Vertrautheit gehören würde. ${ }^{326} \mathrm{Ob}$ weitere Defekte im mentalen Modell für das fehlende Gefühl der Vertrautheit verantwortlich sind (etwa die fehlerhafte oder fehlende Repräsentation charakteristischer Eigenschaften des Partners oder ebensolcher Erinnerungen an gemeinsame Erlebnisse), kann hier offen bleiben, ist aber sicherlich plausibel.

Faktor 2 lässt sich auf die Typ 1-Ableitungen ausgehend vom mentalen Modell beziehen. Ist ein pathologischer Einflussfaktor 2 nicht gegeben, wird das falsche mentale Modell korrigiert. Diese Korrektur erfolgt, wie anzunehmen ist, mittels eines Abgleichs mit anderen mentalen Modellen. So können mentale Modelle aufgrund eines solchen Abgleichs (beispielsweise, propositional paraphrasiert: »Der Partner trägt den gleichen Ehering wie ich, Partner haben gleiche Eheringe, also ist es sehr wahrscheinlich mein Partner «) dazu führen, dass das infragestehende mentale Modell als fehlerhaft zurückgewiesen und somit für weitere Schlussfolgerungen »gesperrt« wird. Ist Faktor 2 gegeben, werden diese Korrekturen blockiert und das Typ 1-Denken nimmt andere Wege: Ausgehend vom fehlerhaften mentalen Modell werden dann Gehalte abgeleitet, die ihrerseits fehlerhaft sind, also fehlerhafte Intuitionen $\mathrm{NP}_{\mathrm{NP}}$. Diese wiederum können die Neigung zu einer intuitiven Überzeugung konstituieren, die dann eine delusionale Überzeugung ist: im Capgras-Beispiel die Überzeugung, dass der Partner durch einen Doppelgänger ersetzt wurde. ${ }^{327}$

326 Die umgangssprachliche Redeweise von >Gefühl soll hier nicht zu wörtlich verstanden werden. Es handelt sich nicht bloß um eine affektive Reaktion beim Anblick des Partners, sondern durchaus um eine Repräsentation: Der Mensch wird als einem nahestehend repräsentiert. Die Repräsentation des Partners als einem nahestehend ist nicht etwa mit der Überzeugung gleichzusetzen, dass der Partner einem nahesteht. Es entspricht dem in dieser Arbeit vertretenen Nonpropositionalismus, dass ich meinen Partner als mir nahestehend repräsentieren kann, ohne dass mir dadurch eine entsprechende Überzeugung zugeschrieben werden muss.

327 Wenn Intuitionen ${ }_{\mathrm{NP}}$ zunächst bloss Neigungen zu Überzeugungen konstituieren (siehe Kap. 3.2) scheint es, als ob hier eine weitere Korrekturmöglichkeit bestünde. »Mein Partner trägt den gleichen Ehering wie ich« könnte also auch als Anfechtungsgrund gelten, der verhindert, dass es zur delusional-intuitiven Überzeugung kommt. In diesem Fall bliebe es aber zumindest bei einer delusionalen `Intuition, dass p $\prec$. Der Fall ist also analog zum Beispiel des Mathematikers, der sich der Spielerfehlschluss-Intuition nicht erwehren kann. Daher scheint es mir nicht zutreffend, an dieser Stelle die Faktor 2- 
Entscheidend ist, dass die delusionalen Vorgänge zunächst auf der Typ 1Ebene, also subpersonal, ablaufen. Das Subjekt muss nicht bewusst von der vorhandenen pathologischen Wahrnehmung auf die Capgras-Delusion schließen. Zudem muss auch die resultierende Delusion nicht auf der Typ 2-Ebene zugänglich sein. Im Fall der Capgras-Delusion ist zwar nur schwer vorstellbar, wie ein derart erschütternder Gedanke (mein Partner wurde durch einen Doppelgänger ersetzt) nicht zugänglich sein und somit nicht als delusionale Überzeugung vorliegen könnte. Doch dies liegt allein an der Dramatik des Inhalts. Auf der theoretischen Ebene spricht nichts dagegen, dass Delusionen auch im Typ 1-Bereich und somit nichtpropositional repräsentiert bleiben können. Im Unterschied zur verbreiteten und im DSM-V festgehaltenen doxastischen Delusions-Konzeption soll daher hier eine nichtdoxastische Konzeption vertreten werden: Delusionen sind nicht einfach eine Form von Überzeugungen, sondern liegen eine Ebene tiefer. ${ }^{328}$ Somit bestehen - analog zu Intuitionen $\mathrm{NP}^{-}$ Delusionen $_{\mathrm{NP}}$, also Gehalte, die entstehen, wenn Subjekte aufgrund neuropsychologischer Pathologien fehlerhafte mentale Modelle erstellen (Faktor 1) oder fehlerhaften Ableitungen aus mentalen Modellen unterliegen (Faktor 2). >Fehlerhaft< kann hier nicht nur heißen, dass eine einfache Täuschung vorliegt, wovon alle Menschen als fallible Erkennende immer wieder betroffen sind, sondern es muss sich um eine massive Abweichung von einer realistischen bzw. rationalen Erkenntnishaltung handeln, die auf Pathologien, d.h. auf feststellbare psychische und/oder neurologische Beeinträchtigungen, zurückführbar ist.

Eine nichtdoxastische und somit nichtpropositionale Intuitionskonzeption lässt sich auch aufgrund der angeführten Beispiele von Delusionen plausibilisieren: Ist es denkbar, dass ein Subjekt den Partner als Doppelgänger, sich selber als tot oder andere Menschen als Verfolger repräsentiert, ohne dass

Korrektur anzusetzen, denn ist eine derartige >Intuition, dass p < gediehen, dürfte bereits diese als Delusion gelten, wenn auch das Subjekt noch nicht eine entsprechende Überzeugung fasst. Eine solche Beschreibung steht allerdings in gewissem Widerspruch zur Beschreibung der Delusion im DSM V, welche diese nur als Überzeugung einordnet. (Diese Konzeption wird im Folgenden aber zurückgewiesen). Es wäre interessant, dem Unterschied zwischen Faktor 2-Korrekturen auf der Ebene der unbewussten Denkprozesse und möglichen solchen Korrekturen auf der Ebene der bewussten Anfechtung von >Intuitionen, dass $\mathrm{p}<$ weiter nachzugehen.

328 Nichtdoxastische Delusions-Konzeptionen vertreten auch McGinn (2004, S. 113-120), Currie (2000) und Currie/Jones (2006). Sowohl bei McGinn als auch bei Currie spielt die Imagination eine entscheidende Rolle. Während McGinn Delusionen als eigenständige mentale Zustände zwischen Überzeugung und Imagination beschreibt, die durch Einflüsse der Imagination auf das Überzeugungssystem zustande kommen, entsteht Currie zufolge eine Delusion dadurch, dass ein Subjekt seine Imaginationen falsch einordnet. 
diese repräsentationalen Gehalte in begrifflicher Form vorliegen müssen (also ohne eine Überzeugung, dass ich verfolgt werde; dass mein Partner durch einen Doppelgänger ersetzt wurde, etc.)? Dies ist ohne Umschweife zu bejahen.

Es bleibt die Frage zu klären, welche der beiden weiter oben erwähnten Möglichkeiten unter der Voraussetzung des skizzierten Delusions- und Intuitionsverständnisses zutrifft: Sind Delusionen eine Subklasse von Intuitionen oder überschneiden sich die beiden Klassen? Wir können die Frage nun präzisieren, indem sie für Delusionen ${ }_{N P}$ und Intuitionen ${ }_{N P}$ gelten soll; denn was auf der propositionalen Ebene gilt, ist gemäß dem hier vertretenen Ansatz nur eine Folge daraus. Die Frage, die hier zu einer Entscheidung führen kann, lautet: Gibt es auch Delusionen ${ }_{\mathrm{NP}}$, die keine Intuitionen $\mathrm{NP}_{\mathrm{N}}$ sind? Falls dies zu bejahen ist, handelt es sich um ein Verhältnis der Überschneidung, falls nicht; sind Delusionen eine Subklasse der Intuitionen.

Um die Frage zu beantworten, kann an die Darstellung des 2-FaktorenAnsatzes erinnert werden. Wenn es zutrifft, dass Delusionen nicht mit pathologischen Wahrnehmungen, sondern vielmehr mit einer pathologisch bedingten Aufrechterhaltung des problematischen Gehalts (wir sprechen nun nicht mehr ausschließlich von delusionalen Überzeugungen) zu tun haben, lässt sich erkennen, dass es auch nichtintuitive Delusionen geben muss. Nehmen wir beispielsweise an, einem Subjekt komme aufgrund einer Pathologie das Gefühl lebendig zu sein abhanden (= >Faktor $1<)$. Nun ist es durchaus möglich, dass sich das Subjekt dieser Wahrnehmung bewusst wird und sie propositional erfasst (»Ich habe das Gefühl, dass ...«). In einem weiteren Akt bewusster Deliberation kann das Subjekt folgendes Argument aufstellen:

(P1) Ich habe das Gefühl, dass ich nicht lebendig bin.

(P2) Wenn man nicht lebendig ist, ist man tot.

$\left(\mathrm{P}_{3}\right)$ Mein Gefühl vermittelt mir die Wahrheit.

(K) Ich bin tot.

Die Pathologie in >Faktor 2< äußert sich dadurch, dass das Subjekt, wie angenommen werden soll, keinen Augenblick an den einzelnen Prämissen dieses Arguments zweifelt und insbesondere jegliche auf die Problematik der dritten Prämisse hinweisende Evidenz völlig ausblendet. Die Pathologie greift im Beispiel ausgehend von einer Wahrnehmungsprämisse auf das deduktive Schließen des Subjekts über. Wir können weiter annehmen, dass der Konklusion >ich bin tot< die phänomenalen Merkmale der Unmittelbarkeit und Gewissheit fehlen - dem Subjekt erscheint unter dieser Annahme die Konklusion nicht als intuitiv. Dennoch fühlt es sich aufgrund seiner Pathologie dazu gezwungen, die Konklusion anzunehmen. Dieser Fall scheint klar möglich zu sein, und es scheint daher passend, dem Subjekt hier eine Delusion zuzuschreiben. Wenn diese Überlegungen zutreffen, wird dadurch erwiesen, 
dass es Fälle nichtintuitiver Delusionen gibt und es sich bei Delusionen daher nicht um eine Subklasse von Intuitionen handeln kann.

Übrig bleibt somit als einzige der Alternativen, dass die Klassen der Intuitionen und Delusionen sich überschneiden. Einige Delusionen, aber nicht alle, sind Intuitionen. Damit eine Delusion auch eine Intuition ist, müssen gemäß den bisherigen Ausführungen zwei Bedingungen erfüllt sein:

(1) Das Subjekt kommt durch Typ 1-Denken ausgehend von mentalen Modellen auf den Gehalt der Delusion ${ }_{\mathrm{NP}}$.

(2) Die Delusion ${ }_{\mathrm{NP}}$ konstituiert eine Neigung zu einer delusionalen Überzeugung, welche (aufgrund der Ableitung aus einem mentalen Modell) die phänomenalen Merkmale der Unmittelbarkeit und Gewissheit aufweist. Damit die Delusion eines Subjekts als Intuition gelten kann, muss also eine Ableitung durch Typ 1-Denken aus einem mentalen Modell erfolgen. Hierbei handelt es sich um eine problematische Ableitung, da sie - wie im 2-Faktoren-Ansatz erklärt - aufgrund einer Pathologie nicht die eigentlich erforderlichen Korrekturen des fehlerhaften mentalen Modells vornimmt. Aus diesen Überlegungen folgt, dass Delusionen nicht eine Subklasse der Intuitionen sind, sondern nur denjenigen Delusionen der Status der Intuition zugesprochen werden sollte, welche die oben genannten Bedingungen erfüllen. Einige, aber nicht alle Delusionen sind Intuitionen; und - wie schon früher festgestellt wurde - einige, aber nicht alle Intuitionen sind Delusionen.

Die Ergebnisse der Untersuchung zum Verhältnis zwischen Intuition und Delusion zusammenfassend lässt sich festhalten, dass es sich aufgrund ihrer Eigenschaften um einander nicht ausschließende, aber auch nicht deckungsgleiche oder hierarchisch geordnete mentale Zustände handelt. Weiter wurde hinsichtlich der Delusionen für die Position des Nondoxastizismus und für den 2-Faktoren-Ansatz plädiert. Von diesen Positionen ausgehend ließ sich nachvollziehen, wie Intuition und Delusion ineinandergreifen können. Eine wichtige Erkenntnis daraus ist, dass Delusionen auch ohne entsprechende Überzeugungen oder gar neben gegenläufigen Überzeugungen bestehen können, wie dies auch bei >Intuitionen, dass $\mathrm{p}<\mathrm{der}$ Fall ist.

Kommen wir mit diesen Resultaten zur Ausgangsfrage zurück: Lässt sich Intuition ausreichend von Delusion abgrenzen, um den epistemischen Status der Intuition zu wahren? Zunächst muss eingeräumt werden, dass die Intuition, wie anhand des 2-Faktoren-Ansatzes gezeigt wurde, ebenso anfällig für pathologisches Denken ist wie andere mentale Zustände. Zur grundsätzlichen Fallibilität der Intuition haben sich also die epistemischen Risiken hinzugesellt, dass unsere Intuition durch motivierte Irrationalität oder pathologisches Denken beeinträchtigt werden kann. Dies bedeutet aber nicht, dass die Grenzen von Intuition zu Delusion (bzw. zuvor: zu Selbsttäuschung und 
Wunschdenken) verwischt würden. Die Schnittmenge zwischen Intuition und Delusion kann bestimmt werden, da genügend klar umrissene Verständnisse von Intuition und Delusion im Hintergrund stehen. Dadurch zeigt sich genauer, in welchen Fällen die Intuitionen Delusionen sind und in welchen nicht. In Bezug auf die Beispiele bedeutet dies: Wir müssen im Extremfall einräumen, dass völlig pathologisch denkende Subjekte (etwa eine verblendete Sektenführerin) über genuine Intuitionen im entsprechenden Bereich verfügen. Durch das genauere Verständnis der Eigenschaften einer Intuition bzw. einer Delusion besteht aber die Möglichkeit, im gegebenen Fall zu untersuchen, ob die Intuition delusional ist oder nicht. Im Übrigen muss wiederholt werden, was bereits als Fazit zum Verhältnis zwischen Intuition und motivierter Irrationalität gesagt wurde: Wenn wir Überzeugungen wegen dieser möglichen Störfaktoren nicht ihren epistemischen Status absprechen (etwa als Grundlagen für Urteile), sollten wir dies auch im Fall der Intuition nicht tun. Es wurde gezeigt, inwiefern Intuitionen gute Gründe für Subjekte sein können; sie sind es nicht immer, wie es auch Überzeugungen nicht immer sind. 